\title{
ARTICLE OPEN Dynamical and thermal magnetic properties of the Kitaev spin liquid candidate $\alpha-\mathrm{RuCl}_{3}$
}

\author{
Pontus Laurell iD $^{1 *}$ and Satoshi Okamoto (iD $^{2 *}$
}

What is the correct low-energy spin Hamiltonian description of $a-\mathrm{RuCl}_{3}$ ? The material is a promising Kitaev spin liquid candidate, but is also known to order magnetically, the description of which necessitates additional interaction terms. The nature of these interactions, their magnitudes and even signs, remain an open question. In this work we systematically investigate dynamical and thermodynamic magnetic properties of proposed effective Hamiltonians. We calculate zero-temperature inelastic neutron scattering (INS) intensities using exact diagonalization, and magnetic specific heat using a thermal pure quantum states method. We find that no single current model satisfactorily explains all observed phenomena of $a-\mathrm{RuCl}_{3}$. In particular, we find that Hamiltonians derived from first principles can capture the experimentally observed high-temperature peak in the magnetic specific heat, while overestimating the magnon energy at the zone center. In contrast, other models reproduce important features of the INS data, but do not adequately describe the magnetic specific heat. To address this discrepancy we propose a modified ab initio model that is consistent with both magnetic specific heat and low-energy features of INS data.

npj Quantum Materials (2020)5:2 ; https://doi.org/10.1038/s41535-019-0203-y

\section{INTRODUCTION}

Quantum spin liquids (QSL) are long-sought-after states of matter without magnetic order, but with nontrivial topological and potentially exotic properties. ${ }^{1,2}$ Much of the search has been focused on frustrated lattice systems, ${ }^{3,4}$ but in an important development in 2006 Kitaev $^{5}$ introduced a novel exactly solvable paradigmatic QSL with bond-directional Ising terms on the bipartite honeycomb lattice. Importantly, this Kitaev model hosts anyonic excitations, ${ }^{6}$ which are of interest both for fundamental reasons and for their proposed application in topological quantum computing. ${ }^{7,8}$ It was realized that such interaction terms naturally appear ${ }^{9}$ - and can be large-in Mott-insulating transition-metal systems with edge-sharing octahedra and strong spin-orbit coupling, such as in $\mathrm{A}_{2} \mid \mathrm{IO}_{3}(\mathrm{~A}=\mathrm{Na}, \mathrm{Li}),{ }^{10,11} a-\mathrm{RuCl}_{3}{ }^{12}$ and other materials. ${ }^{13,14}$

However, the three mentioned materials are all found to order magnetically at low temperatures, and hence cannot be perfect realizations of the Kitaev model. $\mathrm{Na}_{2} \mathrm{IrO}_{3}{ }^{15-17}$ and $a-\mathrm{RuCl}_{3}{ }^{18-21}$ both develop a zigzag order, as illustrated in Fig. $1 \mathrm{a}$, while $\mathrm{Li}_{2} \mid \mathrm{IO}_{3}$ displays an incommensurate spiral order. ${ }^{22}$

Despite the zigzag order, $a-\mathrm{RuCl}_{3}$ has emerged as a particularly promising Kitaev QSL candidate. Initial strong evidence came in the form of a strong and unusually stable scattering continuum at the zone center as observed in inelastic neutron scattering experiments (INS), ${ }^{23-26}$ which has been interpreted as evidence for the presence of fractional Majorana excitations. However, in an alternative picture it has been proposed that the continuum may consist of incoherent excitations due to spontaneous magnon decays. ${ }^{27}$ More recently, half-integer quantization of the thermal Hall conductivity was reported, ${ }^{28}$ also consistent with Majorana excitations. The quantization occurs in a narrow range of in-plane magnetic fields, where the magnetic order is melted, possibly uncovering an intermediate QSL state. ${ }^{24,29-32}$ Further evidence for Kitaev physics has been found in experiments reporting magnetic specific heat, ${ }^{25,33,34} \mathrm{NMR}^{30,35}$ microwave absorption, ${ }^{36}$ Raman scattering, ${ }^{37-39}$ and $\mathrm{THz}$ spectroscopy results. ${ }^{40-43}$

Altogether, these experiments strongly suggest that $a-\mathrm{RuCl}_{3} \mathrm{can}$ be described by a generalized Kitaev-Heisenberg Hamiltonian, ${ }^{13,14}$ including off-diagonal and further-range interactions. Theoretical work leads to the same picture, whether the model is deduced from ab initio methods, ${ }^{12,44-50}$ or from more phenomenological or ab initio-inspired approaches. ${ }^{27,51-53}$ Unfortunately, these different works, as well as experimental fits, ${ }^{26,40}$ have led to a veritable zoo of proposed realistic spin Hamiltonian descriptions for $a-\mathrm{RuCl}_{3}$, and it is not currently clear which description is most accurate. Moreover, the proposed models disagree in terms of included spin-spin interaction terms, magnitudes of interaction parameters, and even signs. We illustrate this situation in Fig. 1c by a scatter plot of the values for just two relevant interaction terms, and in Table 1.

In this work, we adopt a systematic approach to address this uncertainty. We calculate static spin structure factors (SSFs) $S(\mathbf{q})$ and INS intensities $I(\mathbf{q}, \omega)$ for all models listed in Table 1 using Lanczos exact diagonalization (ED) ${ }^{54}$ on 24 -site clusters. We also use ED to explore the evolution of the INS spectra away from the ferromagnetic Kitaev limit as new perturbations are introduced in a generalized Kitaev-Heisenberg model. We then calculate the magnetic specific heat $C_{\text {mag }}$ for the models using the thermal pure quantum state (TPQ) method ${ }^{55}$ on the 24 - and 32-site clusters shown in Fig. 2. A few of the models considered here have previously been studied using similar methods in refs. ${ }^{27,53,56}$. For clarity we will restrict the discussion in the main text to six particularly relevant models. These models all have a ferromagnetic Kitaev coupling $\left(K_{1}<0\right)$, which is expected in $a-\mathrm{RuCl}_{3}{ }^{13,14}$ Results for the other models are included in the Supplementary Information.

Our key finding is that none of the studied models manages to fully capture the salient features of both the INS and magnetic specific heat data. The energy scales obtained in first principles 
(a)

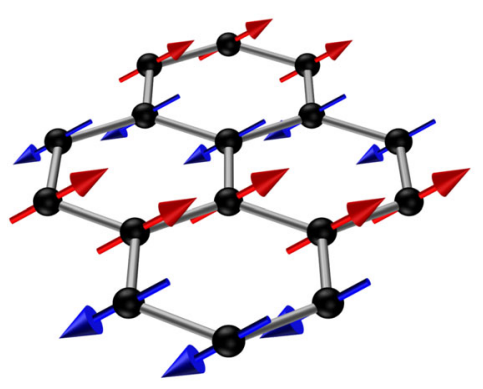

(b)

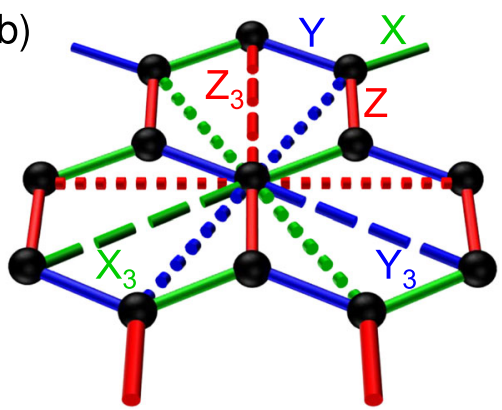

(c)

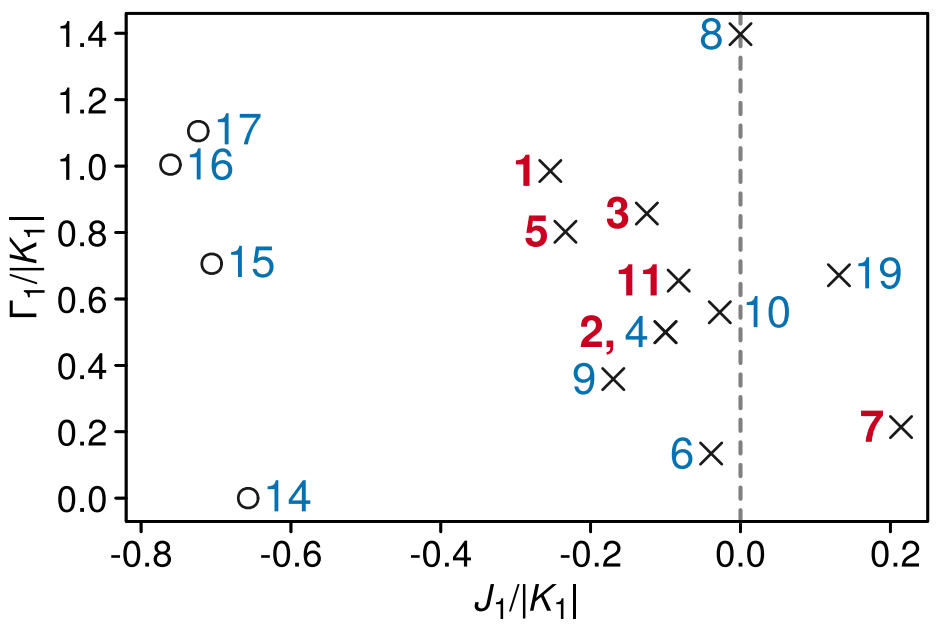

Fig. $1 a-\mathbf{R u C l}_{3}$. a The zigzag magnetic order. $\mathbf{b}$ The honeycomb lattice and its different bonds. Solid, dotted and dashed lines represent nearest, second-nearest and third-nearest-neighbor bonds, respectively. c The variability in two nearest-neighbor (NN) parameters between various proposed spin Hamiltonians for $a-\mathrm{RuCl}_{3}$. The Hamiltonians marked by red, bold numbers (blue, roman) are discussed in the main text (Supplementary Information). Here $K_{1}$ and $J_{1}$ are the NN Kitaev and Heisenberg couplings, respectively, and $\Gamma_{1}$ is an NN symmetric offdiagonal interaction. Models with ferromagnetic (antiferromagnetic) $K_{1}$ are marked with crosses (open circles). Bond-averaged values were used for anisotropic models.

approaches appear to be needed to reproduce a hightemperature peak in $C_{\text {mag }}$, but the parameters proposed in the literature push the INS intensity at the $\Gamma$ point to higher energies. On the other hand, models obtained by fits to INS data run the risk of missing significant off-diagonal interactions, and fail to reproduce the experimentally observed temperature dependence of $C_{\text {mag. }}$. By modifying one of the ab initio models, we are able to find results consistent with both $C_{\text {mag }}$ and low-energy features of the INS spectrum. Our results thus provide important clues for an accurate and realistic description of of $a-\mathrm{RuCl}_{3}$.

\section{RESULTS}

Several of the Hamiltonians listed in Table 1 are special cases of a proposed minimal model ${ }^{13,47}$ for $a-\mathrm{RuCl}_{3}$,

$H_{J_{1}-K_{1}-\Gamma_{1}-J_{3}}=\sum_{\langle i, j\rangle}\left[J_{1} \mathbf{S}_{i} \cdot \mathbf{S}_{j}+K_{1} S_{i}^{\gamma} S_{j}^{\gamma}+\Gamma_{1}\left(S_{i}^{\alpha} S_{j}^{\beta}+S_{i}^{\beta} S_{j}^{a}\right)\right]+J_{3} \sum_{\langle\langle i, j\rangle\rangle\rangle} \mathbf{S}_{i} \cdot \mathbf{S}_{j}$,

where $\langle\ldots\rangle$ and $\langle\langle\langle\ldots\rangle\rangle\rangle$ denote nearest and third-nearest neighbors, respectively. $\gamma=X, Y, Z$ is the bond index shown in Fig. $1 \mathrm{~b}$, and $\alpha, \beta$ are the two other bonds. The $\Gamma_{1}$ term is required to explain the moment direction, and $J_{3}>0$ helps stabilize the zigzag order. $\mathrm{Ab}$ initio and DFT studies also tend to report a sizable symmetric off-diagonal $\Gamma_{1}^{\prime}$ interaction,

$H_{\Gamma_{1}^{\prime}}=\Gamma_{1}^{\prime} \sum_{\langle i, j\rangle} \sum_{a \neq \gamma}\left[S_{i}^{\gamma} S_{j}^{a}+S_{i}^{a} S_{j}^{\gamma}\right]$

which originates from trigonal distortion. ${ }^{57,58}$ Since the crystal structure of $a-\mathrm{RuCl}_{3}$ features trigonal compression ${ }^{13,19,20,25}$ perturbative calculations ${ }^{47,58}$ would predict that $\Gamma_{1}^{\prime}<0$, which provides an additional mechanism to stabilize the zigzag order. ${ }^{58,59}$ In the absence of other crystal distortions, the most general nearestneighbor Hamiltonian would thus be the $J_{1}-K_{1}-\Gamma_{1}-\Gamma_{1}^{\prime}$ model. Combining these two proposed minimal models results in the $J_{1}-K_{1}-\Gamma_{1}-\Gamma_{1}^{\prime}-J_{3}$ model.

Further proposed extensions include second-nearest-neighbor Kitaev and Heisenberg terms, third-nearest-neighbor Kitaev terms, and additional symmetry-allowed anisotropies. ${ }^{44,47}$ In particular, $a-\mathrm{RuCl}_{3}$ does not have a perfect honeycomb lattice, which allows the parameters for the $Z$ bond to deviate from those on the $X, Y$ bonds. In Table 1 we have bond averaged such anisotropies for the sake of clarity, but we will use the full parameter sets in our calculations when appropriate.

Spin structure factors and neutron scattering intensities

Figure 3 shows predicted zero-temperature neutron scattering intensity spectra, $I(\mathbf{q}, \omega)$, for the six central models. All models feature sharp low-frequency peaks at the $M$ points, indicating the zigzag order. The intensity at the $M$ points is significantly higher than the intensity at the $\Gamma$ point, which is inconsistent with experimental observations. ${ }^{24}$ However, the $M$ peaks could potentially be suppressed at finite temperatures. ${ }^{51}$ The models in Fig. 3b-e all show clear signs of the scattering continuum at the $\Gamma$ point at frequencies comparable to the position of the $M$ peak, whereas the two ab initio models in (a) and (f) display a sizable gap up to any noticeable scattering at the $\Gamma$ point. 
Table 1. The spin Hamiltonians for $a-\mathrm{RuCl}_{3}$ considered in this work.

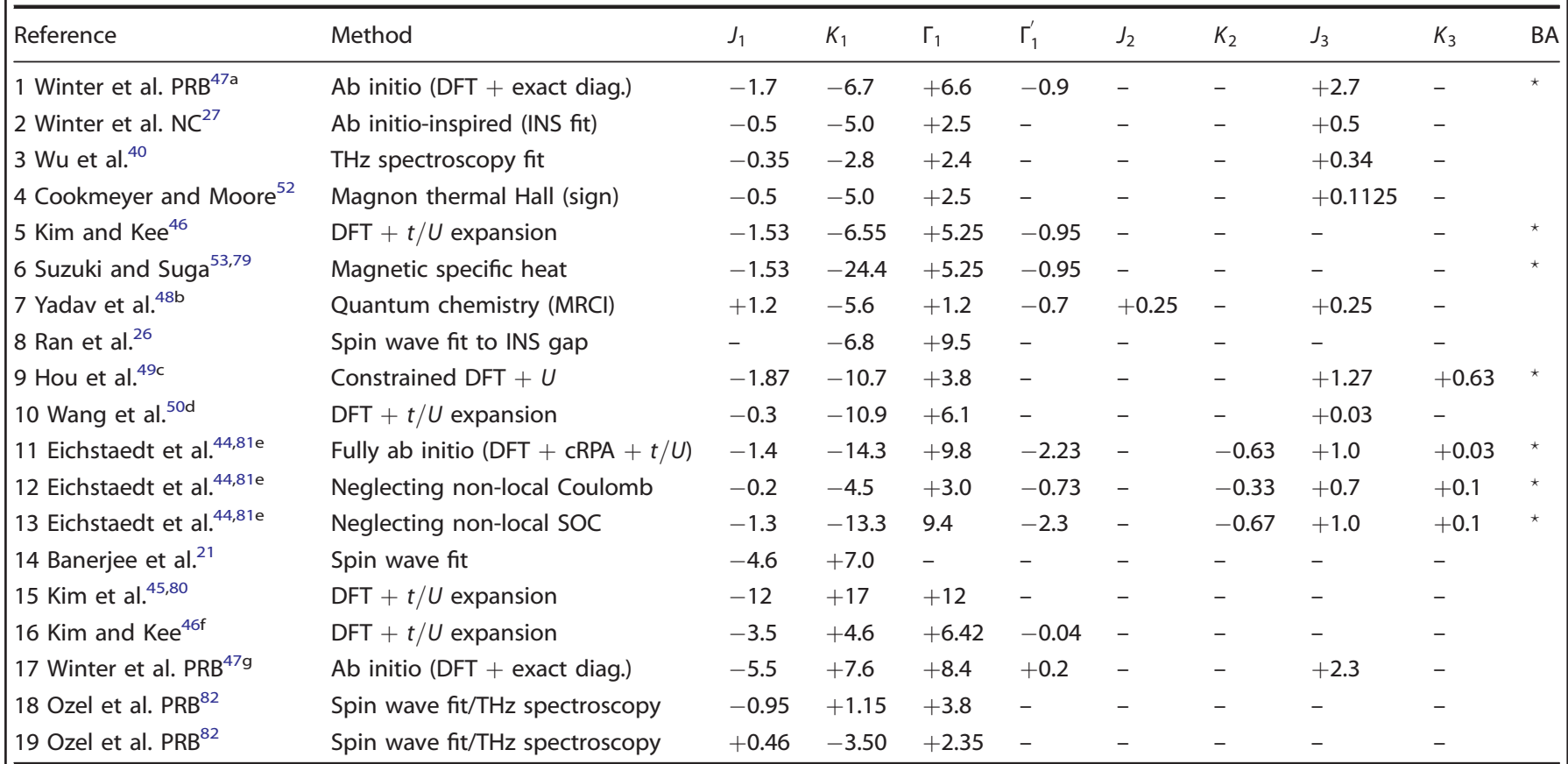

Dashes (-) indicate that the value is unavailable or negligible. The bolded models are considered in the main text, and results for the other models are given in the Supplementary Information. Asterisks in the 'BA' column signify that the full Hamiltonian has different values for the $\mathrm{X} / \mathrm{Y}$ bonds compared with the $\mathrm{Z}$ bonds, and that the parameter values given in the row have been bond averaged

a Using the proposed minimal model, which is bond averaged and neglects small $\Gamma_{1}^{\prime}=-0.9$ meV. Values for the monoclinic ( $\left.C 2 / m\right)$ crystal structure.

${ }^{b}$ We use the sign convention in refs. ${ }^{53,80}$

'This work gives values for several values of $U$. Here we use the $U=3.5 \mathrm{eV}$ parameters

dValues for the C2 structure

'These are the parameters from the preprint version in ref. ${ }^{81}$. They were revised in the published version, ref. ${ }^{44}$. In Supplementary Note 4 we show that this slight modification does not affect our conclusions

${ }^{f}$ Case 0 , corresponding to P3 structure and weaker Hund's coupling than in Model 15

${ }^{9}$ Values for the P3 structure

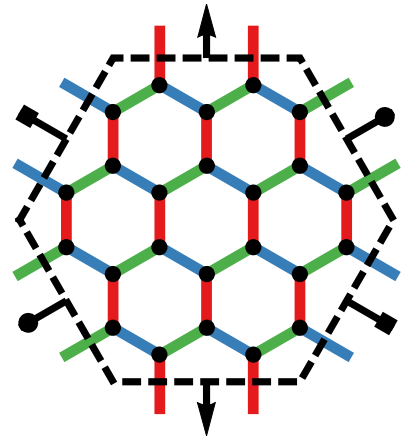

(a) 24-site cluster

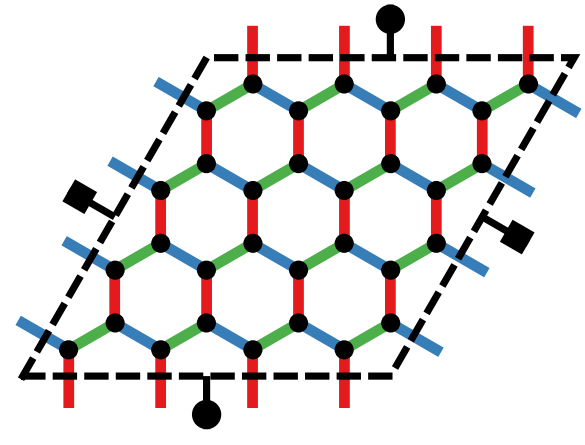

(b) 32-site cluster

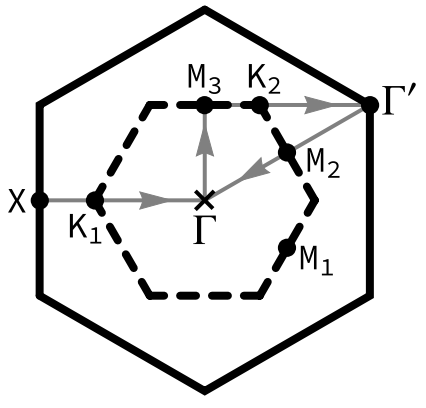

(c) Brillouin zone

Fig. 2 Clusters. a, b Finite size clusters with periodic boundary conditions, and $\mathbf{c}$ the first and second Brillouin zones for the honeycomb lattice. Arrows indicate the high-symmetry path used for $I(\mathbf{q}, \omega)$ spectra.

In the top row of Fig. 4 we have plotted the static spin structure factors $S(\mathbf{q})$. As shown, all six models are consistent with a zigzag ordering with some weight at the zone center. The model shown in Fig. $4 \mathrm{~d}$ showcases how different interaction strengths for the $\mathrm{Z}$ bond results in weakly broken $C_{3}$ symmetry in the structure factors. The bottom three rows of Fig. 4 shows integrated INS intensities for three different energy windows. Experimentally, a star-like pattern with strong weight at $\Gamma$ and arms extending to the $M$ points was observed in the $\omega \in[4.5,7.5]$ meV energy window. ${ }^{23}$
We dub this pattern the M star. The only model displaying this pattern in the right energy window is the one due Yadav et al. ${ }^{48}$, in Fig. 4e. In contrast, (b), (c), and (d) have star-like patterns where the arms extend towards the $\mathrm{K}$ points- $\mathrm{K}$ star shapes. The two ab initio models in (a) and ( $f$ ) do not capture the weight at $\Gamma$ at all, instead forming a flower-like shape that we would expect to see for lower frequencies, since the peak at $\Gamma$ is observed to be higher energy than the $M$ point peak $(2.69 \pm 0.11 \mathrm{meV}$ vs. $2.2 \pm 0.2 \mathrm{meV}$ from INS data. ${ }^{24}$ ) The high-energy window $\omega \in[10.5,20.0] \mathrm{meV}$ is 


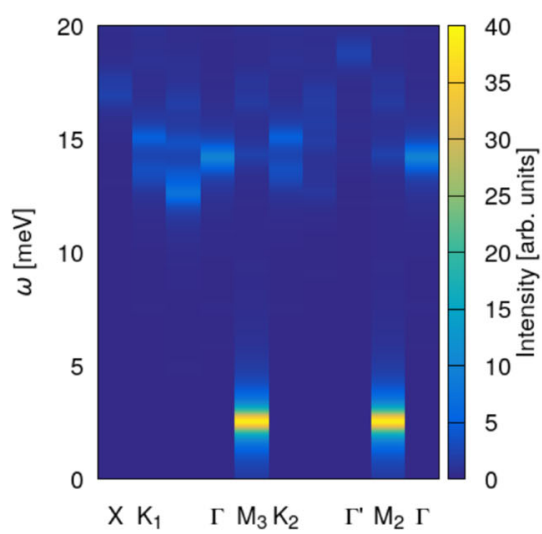

(a) 1. Winter et al. PRB

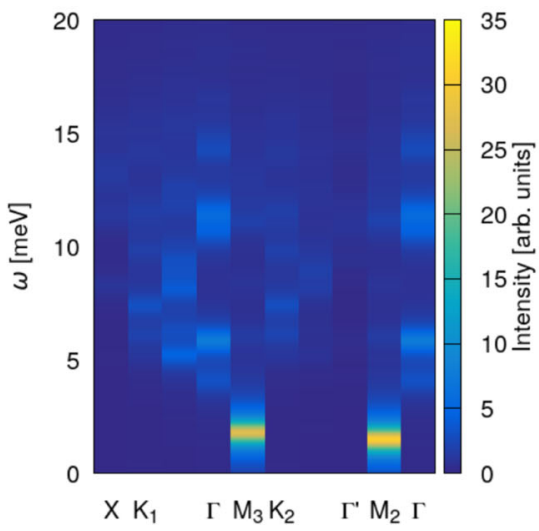

(d) $5 . \mathrm{Kim}$ and Kee

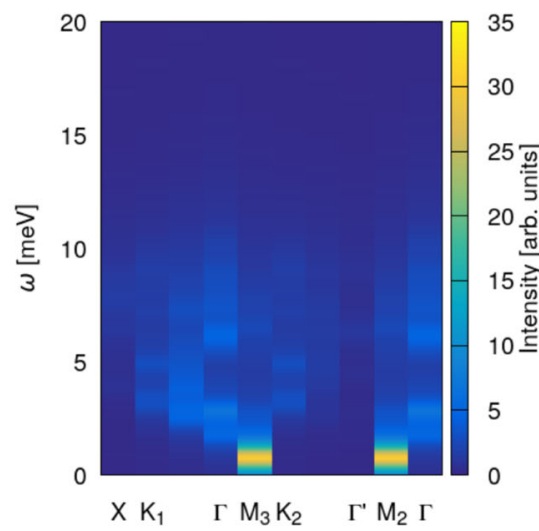

(b) 2. Winter et al. NC

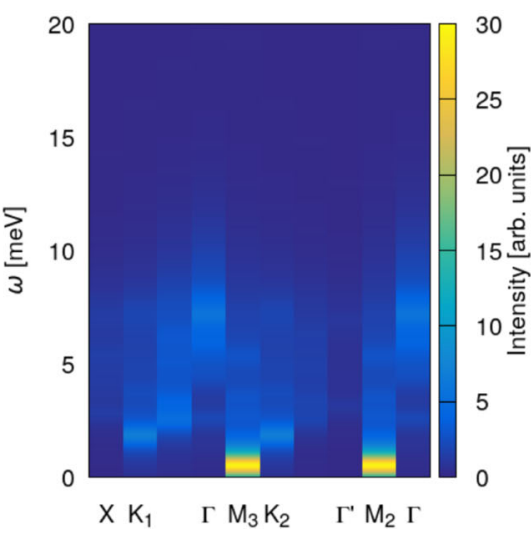

(e) 7. Yadav et al.

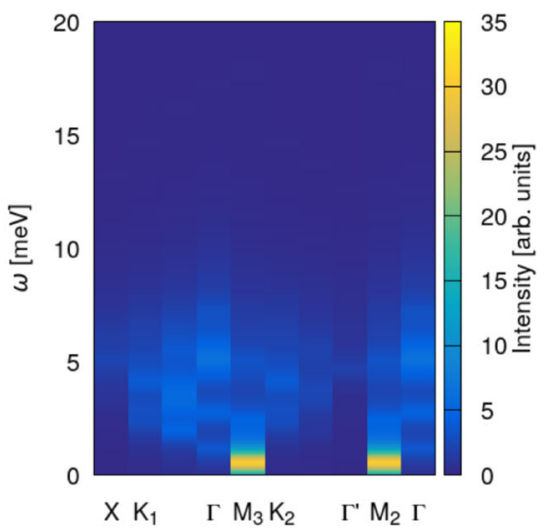

(c) 3. Wu et al.

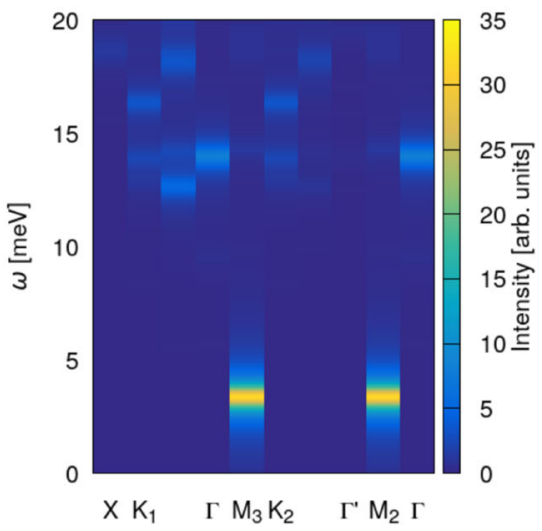

(f) 11 . Eichstaedt et al.

Fig. 3 INS spectra. Inelastic neutron scattering intensities $I(\mathbf{q}, \omega)$ for the chosen models calculated at zero temperature using the $N=24$ site cluster. The model shown in (f) was bond averaged.

expected to be dominated by the continuum at $\Gamma$, which is consistent with (b), (c), (d), (e), but not the "lotus root-like" shapes in (a), and (f).

We summarize our computed neutron scattering intensity results in Table 2, which also includes results for the models discussed in the Supplementary Information. The ab initio-inspired approach of Winter et al. ${ }^{27}$ was constructed to reproduce certain features in the INS spectrum, and thus does particularly well. It reproduces the $\Gamma$ point intensity profile well (see Supplementary Fig. 3), and has an $M$ star shape in the $[5.5,8.5] \mathrm{meV}$ window, but not in $[4.5,7.5] \mathrm{meV}$. It is thus natural to use this model as a starting point for INS data-compatible effective Hamiltonians, as done in the $\mathrm{THz}$ spectroscopy fit of ref. ${ }^{40}$, and an analysis of the magnon thermal Hall conductivity in ref. ${ }^{52}$. The latter work (for results see the Supplementary Note 3) proposes a particularly minor change-only reducing the magnitude of $J_{3}$ from $0.5 \mathrm{meV}$ to $0.1125 \mathrm{meV}$ while keeping other parameters fixed-which actually leads to an $\mathrm{M}$ star shape in the relevant window, but also significantly alters the intensity profile at the $\Gamma$ point. We mention this fact explicitly as an example of a more general observation: for these models even small changes to the parameters can result in significantly different spectra, while even significantly different models can produce very similar SSFs and the same magnetic order. This difficulty calls for other methods to constrain the possible effective Hamiltonians, which is why we will later study the magnetic specific heat.

\section{Evolution of INS spectra}

Above we have provided results for spin Hamiltonians with multiple interaction parameters. To untangle the roles and effects of different interaction terms, we now focus on a minimal
$J_{1}-K_{1}-\Gamma_{1}-\Gamma_{1}^{\prime}-J_{3}$ model. We fix the energy scale $1=\sqrt{J_{1}^{2}+K_{1}^{2}+\Gamma_{1}^{2}+\left(\Gamma_{1}^{\prime}\right)^{2}+J_{3}^{2}}$, and use a hyperspherical parametrization where

$\Gamma_{1}=\cos \theta$

$J_{1}=\sin \theta \cos \phi$

$K_{1}=\sin \theta \sin \phi \cos X$

$\Gamma_{1}^{\prime}=\sin \theta \sin \phi \sin X \cos \psi$,

$J_{3}=\sin \theta \sin \phi \sin \chi \sin \psi$

When $X=0$ this reduces to the notation used in ref. ${ }^{57}$. Following the typical hierarchy of interaction strengths in Table 1 $\left(\left|K_{1}\right| \geq\left|\Gamma_{1}\right| \geq\left|J_{1}\right| \sim\left|\Gamma_{1}^{\prime}\right| \sim\left|J_{3}\right|\right)$ we begin by assuming a dominant FM Kitaev interaction, and introduce other terms one by one. A representative selection of the resulting spectra is shown in Fig. 5. Additional parameter values are studied in Supplementary Note 5.

As Fig. 5a shows, the FM Kitaev limit has a flat spectrum with intensity peaked at the $\Gamma$ point, consistent with the exact theoretical result. ${ }^{60}$ The spectral evolution away from this point can be qualitatively understood using previously obtained phase diagrams. ${ }^{57,58}$ For $\Gamma_{1} / K_{1}=-1 / 2$ shown in Fig. $5 b$, a sharp lowenergy peak develops at the center point between $\Gamma$ and $K_{1}$, " $\mathrm{K}_{1} / 2^{\prime}$, signaling a tendency toward spiral order. The peak at the $\Gamma$ point remains strong, however, preventing a clear signal of the spiral phase in the static spin structure factor. We also note that the resolution of the spiral phase ordering vector is limited by the finite cluster size, and that some zigzag correlations remain 


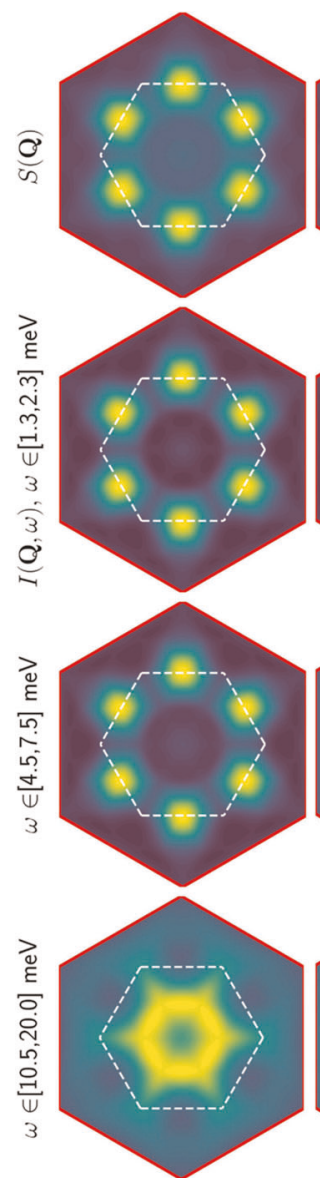

(a) 1. Winter et al. PRB
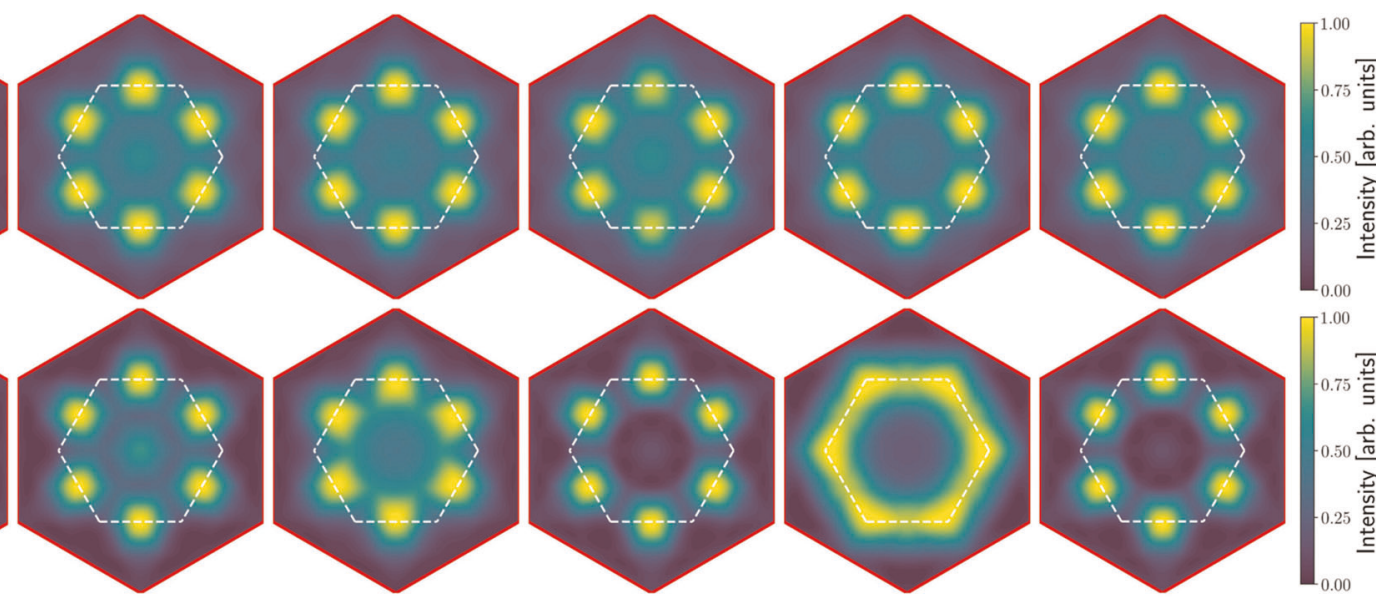

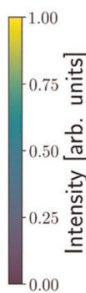
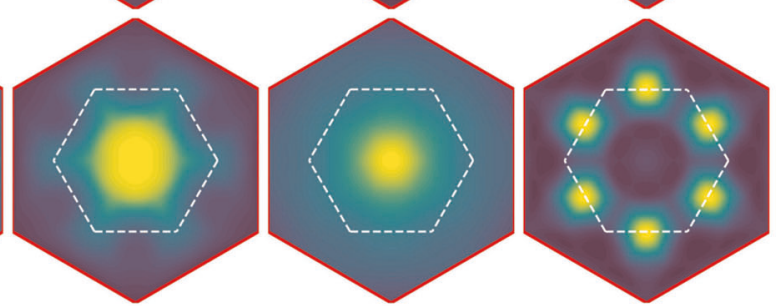

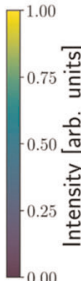

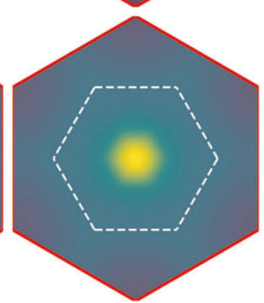

(d) 5. Kim and Kee

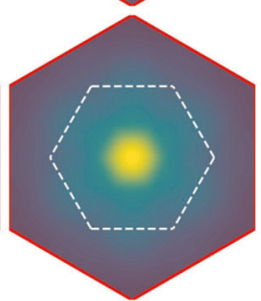

(e) 7. Yadav et al.

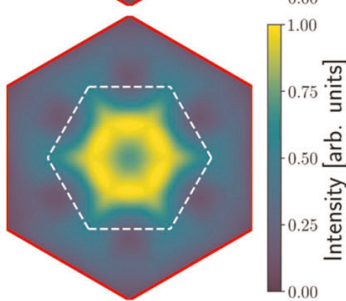

(f) 11. Eichstaedt et al.

Fig. 4 Additional inelastic neutron scattering intensity results obtained on the $N=24$ site cluster. The top row shows the static spin structure factors as a heatmap over $k$-space. The dashed white hexagon marks the first Brillouin zone, and the outer red hexagon shows the second Brillouin zone. The three lower rows show the neutron scattering intensities $I(\mathbf{q}, \omega)$ integrated over representative energy windows (i) $[1.3,2.3] \mathrm{meV}$, (ii) $[4.5,7.5] \mathrm{meV}$, and (iii) $[10.5,20.0] \mathrm{meV}$. Note that each heatmap is normalized separately, in order to showcase patterns in momentum space. Intensities in different heatmaps should not be compared.

at the $M$ points. As $\Gamma_{1}$ is increased to $\Gamma_{1} / K_{1}=-1.0$ in Fig. $5 c$ the intensities at $K_{1} / 2$ and the $M$ points become comparable in strength. As can be seen by comparison with the Kitaev limit, the presence of $\Gamma_{1}>0$ in (b) and (c) tends to produce a stronger excitation continuum, that stretches to higher frequencies.

We next introduce the nearest-neighbor Heisenberg exchange $J_{1}$. Figure $5 d$, e show the effect of adding antiferromagnetic and ferromagnetic $J_{1}$, respectively $\left(J_{1} / K_{1}=\mp 0.1\right)$. In this case $J_{1}>0$ produces a stronger peak at $\mathrm{K}_{1} / 2$ and weaker intensity at the $\Gamma$ point, signaling a stabilized spiral order phase. This is consistent with the classical phase diagram of ref. ${ }^{57}$ and cluster mean field theory results of ref. ${ }^{59}$. In contrast, $J_{1}<0$ sees the peaks at the $\Gamma$ point move down in frequency, a strengthening of the $M$ peaks and significant reduction in intensity at $\mathrm{K}_{1} / 2$. These observations are consistent with moving into the regime of ferromagnetic ordering. We note that a strong continuum remains at the zone center. In the case of $\Gamma_{1} / K_{1}=0$ we would have had a weaker continuum, and a stronger low-energy peak at the zone center. Finally we also introduce $\Gamma_{1}^{\prime}<0$ and $J_{3}>0$ in Fig. 5f, g. These interactions both stabilize the zigzag order, while pushing the $\Gamma$ point peaks to higher frequency, and generally weakening the continuum nature of the excitation spectra. This suggests that the unrealistically large gaps at the $\Gamma$ points in Fig. 3a, $f$ may be due to an overestimation of the $\Gamma_{1}^{\prime}$ and $J_{3}$ parameters.

\section{Magnetic specific heat}

As shown in Fig. 6a, the magnetic specific heat of the pure Kitaev model features two characteristic, well-separated peaks at $T_{1}$ and $T_{\mathrm{h}}$, where the low- $T$ one is due to thermal fluctuations of localized Majorana fermions, and the high- $T$ peak is related to itinerant Majoranas. ${ }^{61,62}$ This two-peak structure appears to be stable to small perturbations away from the Kitaev point. ${ }^{6,63,64}$ Note that the presence of two peaks is not itself a unique signature of Kitaev physics ${ }^{63,65}$ and occurs also for e.g., the $\Gamma$ model, ${ }^{64,66}$ see Fig. 6 a.

A similar two-peak structure has been found in $a-\mathrm{RuCl}_{3}$ experiments, using both $\mathrm{RhCl}_{3}{ }^{34}$ and $\mathrm{ScCl}_{3}{ }^{25,67,68}$ as nonmagnetic analogue compounds. In clean samples, a sharp low- $T$ peak representing the magnetic ordering occurs at $T_{1} \approx 6.5 \mathrm{~K}_{1}^{30,34}$ and then a broader peak occurs at a higher temperature $T_{h}$, followed by a (nonmagnetic) structural transition of $a-\mathrm{RuCl}_{3}$ near $165-170$ $\mathrm{K}^{25,34}$ So far, there is no clear consensus for the precise value of $T_{\mathrm{h}}$ (Widmann et al. report $T_{\mathrm{h}} \approx 70 \mathrm{~K}^{34}$ Do et al. find $T_{\mathrm{h}} \simeq 100 \mathrm{~K}_{1}^{25}$ while Hirobe et al. ${ }^{67}$ and Kubota et al. ${ }^{68}$ find a broad maximum around $80-100 \mathrm{~K}$ ), but it appears to be an order of magnitude larger than $T_{\mathrm{I}}$. Whether or not the $T_{\mathrm{h}}$ peak can be attributed to fractionalized excitations due to a proximate Kitaev QSL, the feature appears to be real and ought to be captured by a realistic spin Hamiltonian. Two additional comments are in order. First, accurately determining the magnetic specific heat at higher temperatures is challenging, and sensitive to details of the 
Table 2. Summary of results, highlighting important features in the INS and magnetic specific heat predictions on the 24-site cluster.

\begin{tabular}{|c|c|c|c|c|}
\hline Reference & $\omega_{\ulcorner}[\mathrm{meV}]$ & $\omega_{\mathrm{M}}[\mathrm{meV}]$ & IM shape & $T_{\mathrm{h}}[\mathrm{K}]$ \\
\hline 1 Winter et al. PRB ${ }^{47}$ & 14.2 & 2.55 & Flower & 54 \\
\hline 2 Winter et al. $\mathrm{NC}^{27}$ & 2.8 & 0.75 & K star & 22 \\
\hline $3 \mathrm{Wu}$ et al. ${ }^{40}$ & 1.18 & 0.54 & Dominated by $\Gamma$ & 17 \\
\hline $\begin{array}{l}4 \text { Cookmeyer and } \\
\text { Moore }^{52}\end{array}$ & $1.67^{\mathrm{a}}$ & 0.57 & M star & 24 \\
\hline $5 \mathrm{Kim}$ and $\mathrm{Kee}^{46}$ & 4.12 & 1.49 & K star-like & 34 \\
\hline $\begin{array}{l}6 \text { Suzuki and } \\
\text { Suga }^{53,79}\end{array}$ & 2.05 & 1.98 & K star & 94 \\
\hline 7 Yadav et al. ${ }^{48}$ & 2.58 & 0.58 & M star & 13 \\
\hline 8 Ran et al. ${ }^{26}$ & 4.8 & 1.37 & M star & 57 \\
\hline 9 Hou et al. ${ }^{49}$ & 5.5 & 1.87 & K star & 33 \\
\hline 10 Wang et al. ${ }^{50}$ & $3.13^{\mathrm{a}}$ & 1.03 & K star & 53 \\
\hline 11 Eichstaedt et al. ${ }^{44}$ & 11.2 & 2.79 & Flower $/ \mathrm{M}_{3}{ }^{\mathrm{b}}$ & 66 \\
\hline 12 Eichstaedt et al. ${ }^{44}$ & 5.1 & 0.98 & Lotus root & 22 \\
\hline 13 Eichstaedt et al. ${ }^{44}$ & 11.9 & 2.43 & Dominated by $\mathrm{M}_{3}$ & 63 \\
\hline 14 Banerjee et al. ${ }^{21}$ & 6.38 & 1.21 & Lotus root & 21 \\
\hline 15 Kim et al. ${ }^{45,80}$ & 6.52 & 2.65 & Flower & 81 \\
\hline $16 \mathrm{Kim}$ and $\mathrm{Kee}^{46}$ & 4.39 & 2.25 & $\begin{array}{l}\text { Dominated by } \\
\Gamma \text { and } K\end{array}$ & 35 \\
\hline 17 Winter et al. PRB ${ }^{47}$ & 7.35 & 0.43 & Ring & 41 \\
\hline 18 Ozel et al. PRB ${ }^{82}$ & 4.39 & 0.31 & Dominated by $\Gamma$ & 16 \\
\hline 19 Ozel et al. PRB ${ }^{82}$ & 3.18 & 3.92 & Dominated by $\Gamma$ & 18 \\
\hline \multicolumn{5}{|c|}{$\begin{array}{l}\text { We focus on (i) the positions } \omega_{\Gamma} \text { and } \omega_{M} \text { of the initial spin wave peaks in the } \\
\text { INS intensity at the } \Gamma \text { and } M \text { points, respectively, (ii) the shape of the } \\
\text { neutron scattering intensity map (IM) in momentum space integrated over } \\
{[4.5,7.5] \text { meV, and (iii) the position of the high-temperature peak in the }} \\
\text { magnetic specific heat. } K(M) \text { star denotes a star-like shape pointing } \\
\text { towards the } K(M) \text { points. The bold models are considered in the main text. } \\
\text { Results for the other models are given in the Supplementary Information } \\
\text { a There is also a clear, distinct peak at lower frequency, which would be } \\
\text { hidden by the elastic scattering continuum } \\
{ }^{b} \text { Flower shape when bond-averaged, otherwise dominated by } M_{3} \text {. Peak } \\
\text { positions are given for the non-bond-averaged case }\end{array}$} \\
\hline
\end{tabular}

analysis. This may partly explain the range of $T_{\mathrm{h}}$ values mentioned above. Second, an optical spectroscopy study ${ }^{69}$ extracted a crossover temperature for magnetic correlations, $T^{\star} \sim 35 \mathrm{~K}$, which, in an analysis relying on the pure Kitaev model, was equated to $T_{\mathrm{h}}$. In the following we will rely mainly on data from Widmann et al., ${ }^{34}$ but there is clearly some uncertainty to the value of $T_{\mathrm{h}}$.

In Fig. 6b, c we plot the magnetic specific heat, $C_{\text {mag }}(T)$, for the six considered models on 24-site clusters, along with the excess heat capacity determined in ref. ${ }^{34}$. We note that the finite-size clusters are far from the thermodynamic limit, so we cannot expect to numerically observe a sharp magnetic transition, but the location of the peaks can provide useful information. We see that the models plotted in (b) are clearly inconsistent with the experimental data. However, the two ab initio models in (c) (which did not capture the INS data) actually have peak positions that are consistent with the data. In fact, the model fully determined from first-principles in Eichstaedt et al. ${ }^{44}$ has a peak at $T_{\mathrm{h}} \approx 66 \mathrm{~K}$, while the experimental data are centered around $70 \mathrm{~K}$, with a peak at $68 \mathrm{~K}$.

In Fig. 7 we provide 32-site cluster TPQ results for a subset of the models, and show the finite-size scaling tendencies. The two cluster sizes have different symmetry properties, which could explain part of the differences. Unfortunately, going to even larger cluster sizes (for better scaling or to preserve symmetries) using the TPQ method becomes computationally prohibitive. We find that the position of the high-temperature peak changes only marginally (see Supplementary Note 2 for details), while the lowtemperature behavior is much less well-converged. We thus conclude that the two ab initio models describe the magnetic specific heat better than the other models.

Modified ab initio model

Having established that the two ab initio models are consistent with the experimental specific heat, we now ask whether they can be modified to better describe the INS data. As discussed in the section on evolution of INS spectra, the large gaps at the zone centers in Fig. $3 a$, $f$ may be due to overestimated $\Gamma_{1}^{\prime}$ or $J_{3}$ values. Since $\Gamma_{1}^{\prime}$ is sensitive to the degree of trigonal distortion, it can be expected to vary between crystal samples. It is thus likely the parameter with the highest degree of uncertainty. For these reasons, we consider the effect of reducing $\left|\Gamma_{1}^{\prime}\right|$ in the model of ref. ${ }^{44}$, while leaving other parameters ( $J_{3}$ included) unchanged. We use bond-averaged interaction parameters.

We again find that the spin wave gap at the $\Gamma$ point depends strongly on the value of $\Gamma_{1}^{\prime}$, and that the low-energy features of the INS spectrum can be well explained when $\left|\Gamma_{1}^{\prime}\right|$ is significantly reduced but still finite. Specifically, we take $r_{1}^{\prime} \rightarrow 0.05 r_{1}^{\prime}$. (The full parameter set is given in Supplementary Table 2.) The spectrum for this case is shown in Fig. 8a. In this case, we find $\omega_{\Gamma}=2.5 \mathrm{meV}$, $\omega_{M}=2 \mathrm{meV}$, close to the values obtained in ref. ${ }^{24}: \omega_{\Gamma}=2.69 \pm 0.11$ $\mathrm{meV}, \omega_{\mathrm{M}}=2.2 \pm 0.2 \mathrm{meV}$. We note that our value for $\omega_{\Gamma}$ is consistent with the the low-energy magnon energy of $2.5 \mathrm{meV}$ observed using $\mathrm{THz}$ spectroscopy. ${ }^{40,41}$ As we noted earlier, the relative intensity of the $\Gamma$ point peak may be enhanced at finite temperature. ${ }^{51}$

Figure $8 \mathrm{~b}$ shows the SSF, which remains consistent with zigzag order, and $I(\mathbf{q}, \omega)$ integrated over the $[4.5,7.5] \mathrm{meV}$ range. From this integrated intensity and Fig. 8 a we see that there is a lack of intensity at the zone center within the chosen energy range, unlike the notable star shape in ref. ${ }^{23}$. The source of this discrepancy is not clear, and calls for further study and parameter refinement. Finally, in (c) we show the magnetic specific heat calculated for this modified model. We do find $T_{\mathrm{h}} \approx 83 \mathrm{~K}$, which is higher than the $\approx 70 \mathrm{~K}$ reported in ref. ${ }^{34}$, yet consistent with the broader range of values proposed (70-100 K).

\section{DISCUSSION}

We have found that there is a considerable qualitative difference between proposed spin Hamiltonians that describe the INS data well, and realistic models derived using ab initio methods, which are consistent with the reported magnetic specific heat observations. This difference is accompanied by a significant discrepancy in overall energy scales. The specific heat measurements probe the energy density of states, and should represent a good guide to the energy scale, provided the phonon background is handled adequately. In light of our results we thus expect the Kitaev and off-diagonal couplings strengths to be larger, and that $a-\mathrm{RuCl}_{3}$ may be closer to the QSL regime than previously believed. (We note that recent anisotropic susceptibility ${ }^{70}$ and $\mathrm{THz}$ spectroscopy experiments ${ }^{41}$ are also consistent with higher Kitaev strengths than in Model 2.) In contrast, the calculated dynamical spin structure factors and INS intensities are much more sensitive to the relative strengths of different interaction terms. They are particularly useful probes for models with fewer degrees of freedom, such as the $J_{1}-K_{1}-\Gamma_{1}-\Gamma_{1}^{\prime}-$ $J_{3}$ model we study. At the same time, static properties such as magnetic order or SSFs, are clearly insufficient to fully constrain the $a-\mathrm{RuCl}_{3}$ spin Hamiltonians. In this respect, properties in the presence of magnetic fields, such as phase transitions and the 


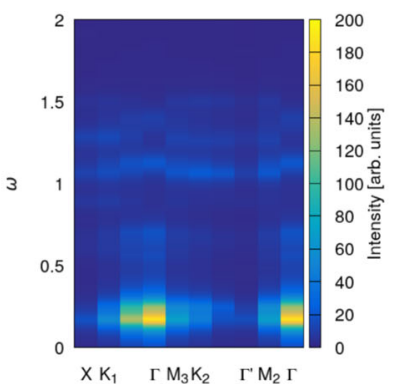

(a) FM Kitaev limit, $K_{1}=-1$

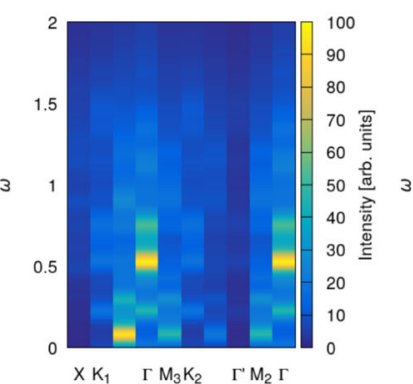

(b) $\Gamma_{1} / K_{1}=-0.5$

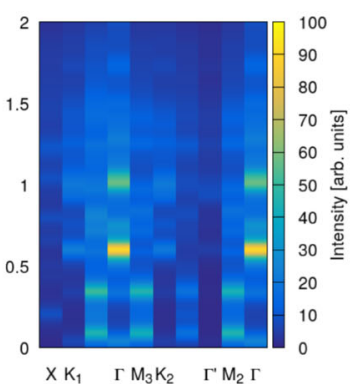

(c) $\Gamma_{1} / K_{1}=-1.0$

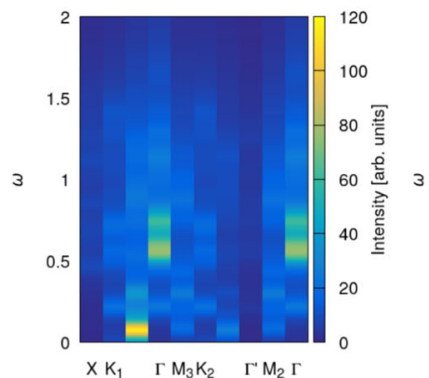

(d) $\Gamma_{1} / K_{1}=-0.5, J_{1} / K_{1}=-0.1$

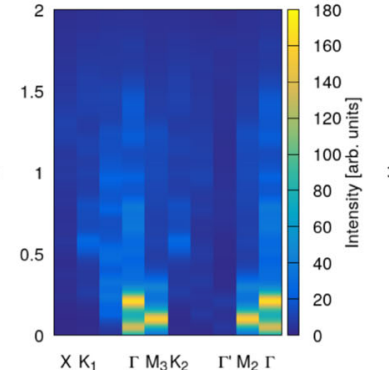

(e) $\Gamma_{1} / K_{1}=-0.5, J_{1} / K_{1}=+0.1$

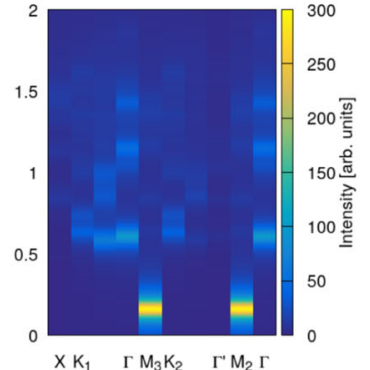

(f) $\Gamma_{1} / K_{1}=-0.5, J_{1} / K_{1}=+0.1$, $\Gamma_{1}^{\prime} / K_{1}=+0.15$

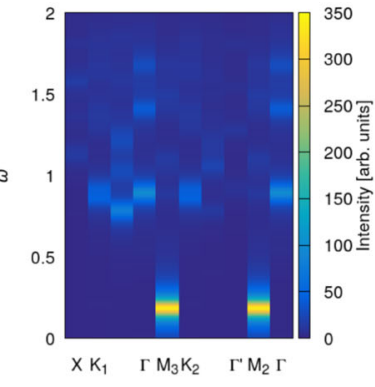

(g) $\Gamma_{1} / K_{1}=-0.5, J_{1} / K_{1}=+0.1$, $\Gamma_{1}^{\prime} / K_{1}=+0.15, J_{3} / K_{1}=-0.1$

Fig. 5 Evolution of the INS intensity $I(\mathbf{q}, \omega)$ away from the ferromagnetic Kitaev limit. The limit is shown in $\mathbf{a}$. In $\mathbf{b}, \mathbf{c}$ a $\Gamma_{1}>0$ term is added. The cases of AFM $J_{1}$ and FM $J_{1}$ are considered in $\mathbf{d}$, e, respectively. Finally $\Gamma_{1}^{\prime}<0$ and $J_{3}>0$ are introduced in $\mathbf{f}$, $\mathbf{g}$. These results were obtained using the 24-site cluster.

magnon thermal Hall effect, ${ }^{52}$ present a particularly promising direction for both theory and experiment.

By using one of the ab initio models (Eichstaedt et al. $^{44}$ ) as a starting point and reducing the magnitude of $\Gamma_{1}^{\prime}$, we are able to identify a set of parameters (full values are given in Supplementary Table 2) that partly resolve the discrepancy between the two classes of models mentioned above. We find low-energy peaks in the INS spectrum and a high-temperature peak in the magnetic specific heat that are consistent with experiment. However, this should not yet be considered a fully accurate model, as there is an unexplained lack of intensity at the zone center at intermediate frequencies. Instead, we consider it a new starting point.

With these results in mind, we now return to the question we posed in the abstract, about the nature of the correct spin Hamiltonian for $a-\mathrm{RuCl}_{3}$. From a variety of ab initio and DFT calculations, we expect a minimal model to include ferromagnetic nearest-neighbor Kitaev and Heisenberg couplings, $\Gamma_{1}>0, a \Gamma_{1}^{\prime}<0$ term, and a small $J_{3}>0$. Our results for the modified ab initio model further suggest that $\Gamma_{1}^{\prime}$ should be small, but finite. Since both $\Gamma_{1}^{\prime}$ and $J_{3}$ act to stabilize the zigzag order, small values are consistent with the fact that a relatively weak in-plane magnetic field can take $a-\mathrm{RuCl}_{3}$ out of the ordered phase. Alternatively, $a-\mathrm{RuCl}_{3}$ might be close to a quantum critical point, ${ }^{32,33,51}$ which would be a very exciting scenario. Anisotropic susceptibility measurements ${ }^{70}$ point towards significant off-diagonal $\Gamma_{1}$ and $\Gamma_{1}^{\prime}$ terms, which may also help stabilize the purported spin liquid phase at finite magnetic fields. ${ }^{6471,72}$ At this point it is not clear whether anisotropies between bonds or the interlayer coupling play a qualitative role, but they are also expected in a full model.

We hope that our results can help guide further theory development and interpretation of experimental results going forward, both for $\mathrm{a}-\mathrm{RuCl}_{3}$ and other Kitaev spin liquid candidate materials. Since we found the INS predictions to be particularly sensitive to small parameter changes, it would be very useful to consider additional modeling techniques and additional observables. For example, machine learning methods may be a promising way to efficiently handle the high-dimensional parameter space. In addition, further experiments in applied magnetic fields can help constrain the Hamiltonian by suppressing fluctuations.

\section{METHODS}

We use the $\mathcal{H} \Phi^{73}$ library for numerical calculations on finite-size systems. We employ a 24-site cluster with $C_{3}$ symmetry, and a rhombic 32-site cluster, see Fig. 2. The momenta compatible with the finite-size clusters are shown in Supplementary Note 1. Finite-temperature specific heat is computed using the microcanonical thermal pure quantum state (TPQ) method, ${ }^{55,63,74}$ and averaged over $\geq 15$ random initial vectors. The key idea behind the TPQ method is that a quantum system at thermal equilibrium can be reliably described by a single, iteratively constructed state. Utilizing this fact allows for a significant reduction in computational cost compared with finite-temperature exact diagonalization methods.

Zero-temperature properties are calculated using the Lanczos exact diagonalization method, and the continued fraction expansion (CFE) ${ }^{54}$ is used to compute the dynamical quantities. A total of 500 Lanczos steps are used to calculate the CFE. We take $1 \mathrm{meV}$ as a representative value for the experimental energy resolution at full-width/half-maximum, 23,24 and emulate it in the exact diagonalization calculations by using a Lorentzian broadening of $0.5 \mathrm{meV}$. For the INS spectral evolution calculations we used a Lorentzian broadening of 0.05 in the fixed energy scale. The neutron scattering intensity $I(\mathbf{q}, \omega)$ is defined ${ }^{27,51}$

$I(\mathbf{q}, \omega) \propto f^{2}(q) \sum_{\mu, \nu}\left[\delta_{\mu \nu}-\frac{q_{\mu} q_{\nu}}{q^{2}}\right] S^{\mu \nu}(\mathbf{q}, \omega)$,

where $f(q)$ is the magnetic form factor, $q^{a}$ is the projection of the momentum vector onto the spin components in the local cubic coordinate system also used for the spin Hamiltonian, and $S^{\mu \nu}(\mathbf{q}, \omega)$ is the dynamical spin structure factor at momentum $\mathbf{q}$ and frequency $\omega$,

$$
S^{\mu \nu}(\mathbf{q}, \omega)=\int \sum_{i, j}\left\langle S_{i}^{\mu}(t) S_{j}^{\nu}(0)\right\rangle \mathrm{e}^{-i \mathbf{q} \cdot\left(\mathbf{r}_{i}-\mathbf{r}_{j}\right)} \mathrm{e}^{-i \omega t} \mathrm{~d} t
$$

Note that the off-diagonal elements of $S^{\mu \nu}(\mathbf{q}, \omega)$ contribute significantly for most models studied here, due to the presence of $\Gamma_{1}$ and $\Gamma_{1}^{\prime}$ interactions. 


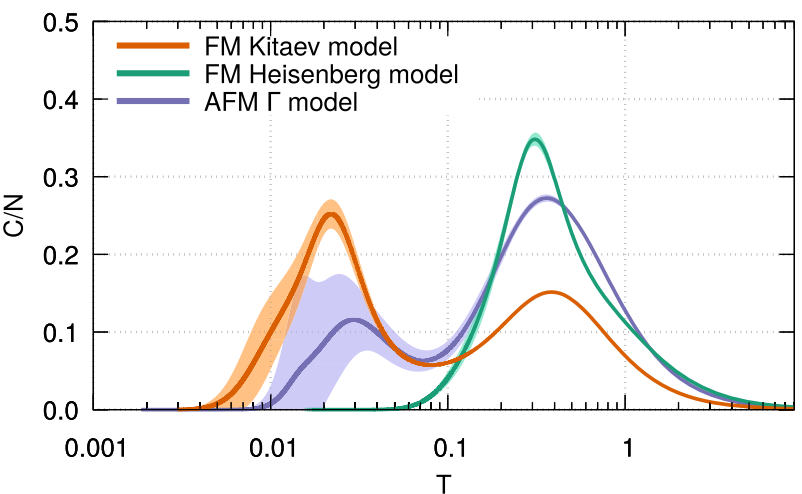

(a) The Kitaev, Heisenberg and Gamma models.

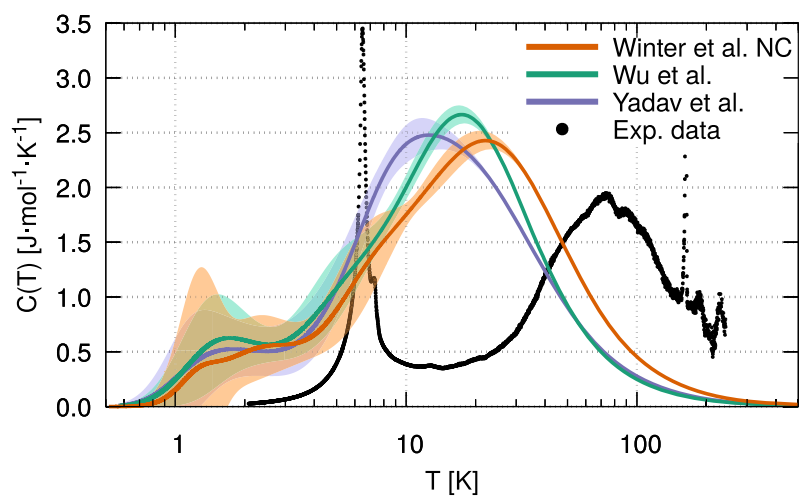

(b) Models 2, 3, and 7 .

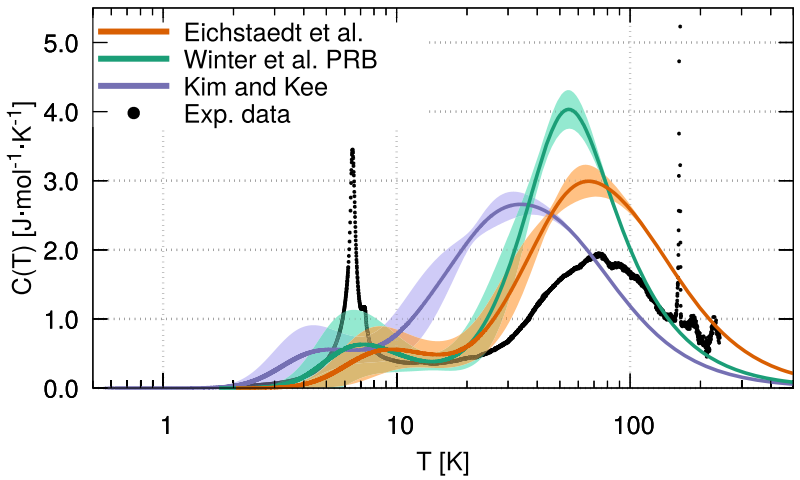

(c) Models 1, 5, and 11 .

Fig. 6 Magnetic specific heat calculated using the TPQ method and the $N=24$ site cluster. The solid lines show the average value over 15 initial vectors ( 100 vectors were used for the $\Gamma$ model), and the shaded areas show the standard deviation. a shows $C_{\text {mag }}$ for the ferromagnetic Kitaev $\left(K_{1}=-1\right)$, ferromagnetic Heisenberg $\left(J_{1}=-1\right)$, and "antiferromagnetic" Gamma $\left(\Gamma_{1}=+1\right)$ models. b, c show $C_{\text {mag }}$ for the six chosen models for $a-\mathrm{RuCl}_{3}$. For comparison, the experimentally determined excess heat capacity from ref. ${ }^{34}$ is plotted using black dots. The peak in the experimental data near $6.5 \mathrm{~K}$ signals the magnetic ordering, and the strong peak at $170 \mathrm{~K}$ is a structural transition, unrelated to the magnetic specific heat. Finally, the peak near $70 \mathrm{~K}$ may correspond to itinerant Majorana quasiparticles. ${ }^{34,61,62}$ The peak position is inconsistent with the models plotted in $\mathbf{b}$, but consistent with the ab initio models plotted in c, with higher interaction strengths.

The static spin structure factor, $S(\mathbf{q})=\int S(\mathbf{q}, \omega) \mathrm{d} \omega$, is evaluated separately. We note that neutron scattering experiments probe the magnetization $\mathbf{M}$, while the Hamiltonians in Eqs. (1) and (2) are expressed in terms of the pseudospin S. Hence the form of Eq. (9) amounts to an assumption that $\mathbf{M}$ and $\mathbf{S}$ are approximately parallel. However, trigonal

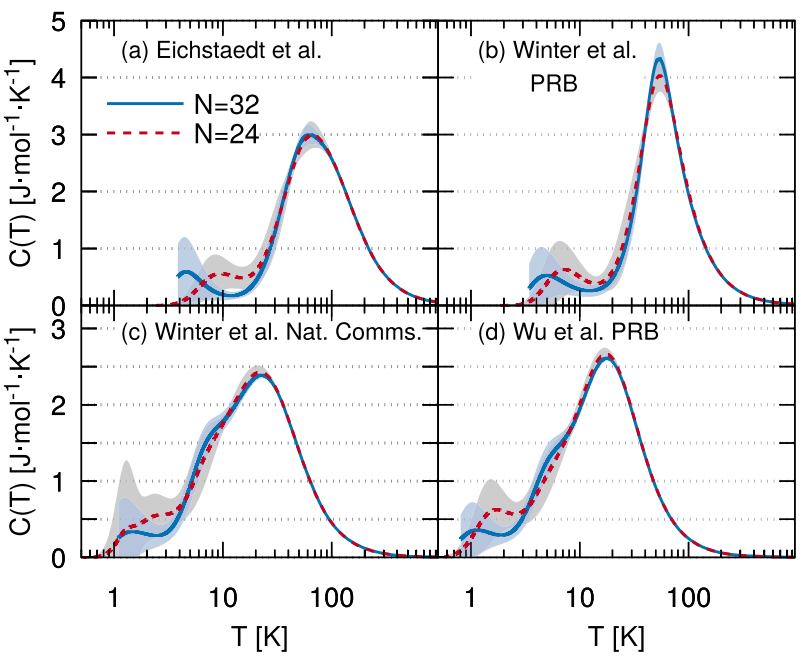

Fig. 7 Finite size scaling of specific heat for four select Hamiltonians (models 1, 2, 3, and 11). The data are averaged over 15 initial TPQ vectors, and the shaded regions show the standard deviations. We generically observe a two-peak structure, the high- $T$ peak of which appears to be well converged. The lower temperature peak corresponds to magnetic ordering, and may be quite sensitive to finite-size effects, or to the difference in symmetry between the 24- and 32-site clusters.

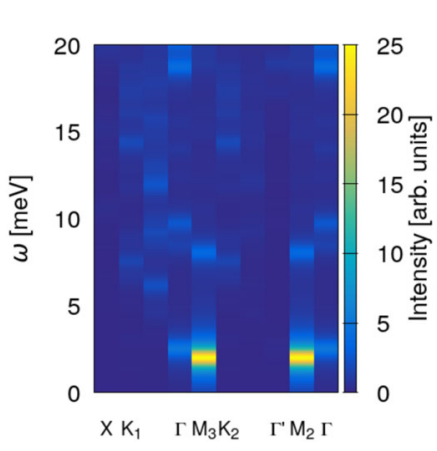

(a) INS spectrum

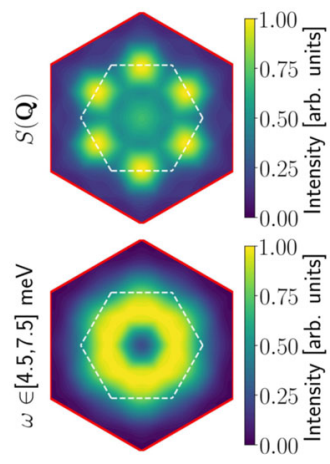

(b) SSF and integrated $I(\mathbf{q}, \omega)$

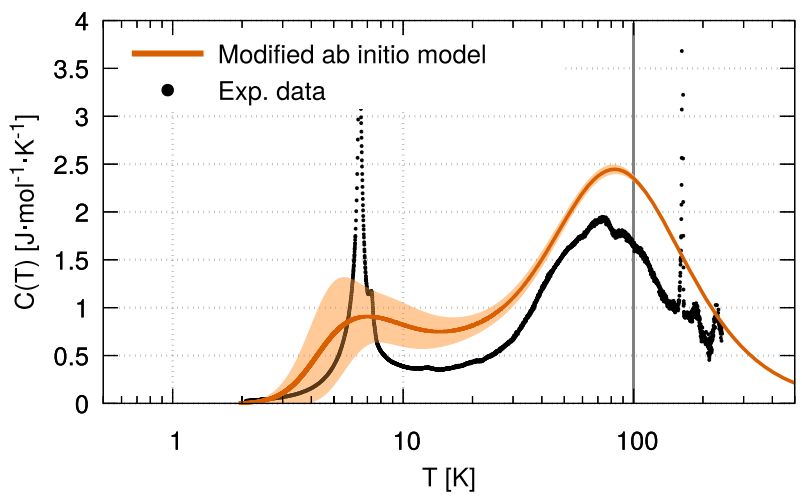

(c) Magnetic specific heat

Fig. 8 The modified ab initio model. Shown in a is the $I(\mathbf{q}, \omega)$ spectrum for the modified ab initio model, calculated using ED. We find low-energy peaks at the $M$ and $\Gamma$ points consistent with peaks in experimental INS data. The SSF is shown in the top panel of $\mathbf{b}$, clearly consistent with a zigzag order. The bottom panel of $\mathbf{b}$ shows $I(\mathbf{q}, \omega)$ integrated over $[4.5,7.5] \mathrm{meV}$, with less intensity at the zone center than is experimentally observed. c shows the magnetic specific heat calculated using TPQ and 15 random initial vectors compared with the experimental data from ref. ${ }^{34}$. The shaded region shows the standard deviation. The calculations were all done using the 24-site cluster. 
distortion can induce an angle between the two vectors, and a resulting $g$-factor anisotropy. ${ }^{75}$ While there have been conflicting reports about the degree of anisotropy, ${ }^{48,68,76}$ a more recent $X$-ray absorption spectroscopy study ${ }^{77}$ found $a-\mathrm{RuCl}_{3}$ to have only weak trigonal distortion and a nearly isotropic $g$-factor. In light of this result and the relatively weak $\Gamma_{1}^{\prime}$ interactions in Table 1 we assume that $\mathbf{M}$ and $\mathbf{S}$ are indeed approximately parallel in $a-\mathrm{RuCl}_{3}$.

$f(q)$ is assumed to be isotropic, which is justified for small scattering wave numbers. ${ }^{23}$ The magnetic form factor for $\mathrm{Ru}^{3+}$ was calculated using DFT in the Supplementary Material of ref. ${ }^{25}$. By fitting their data to a Gaussian we have obtained the analytical approximation

$f(q)=\exp \left(-\frac{q^{2}}{(2 \pi * 0.25)^{2}}\right)$.

We integrate over the momentum direction perpendicular to the honeycomb plane, following the experiment. ${ }^{23}$ Since the ED calculation is necessarily two-dimensional we assume that $S^{\mu \nu}(\mathbf{q}, \omega)$ is constant along the perpendicular direction during the integration step. We expect this to be a reasonable approximation due to the strong two-dimensionality of $a-\mathrm{RuCl}_{3}{ }^{78}$ and the relatively small interlayer coupling. ${ }^{32}$

\section{DATA AVAILABILITY}

The data that support the findings of this study are available from the corresponding author upon reasonable request.

Received: 18 June 2019; Accepted: 16 December 2019; Published online: 10 January 2020

\section{REFERENCES}

1. Savary, L. \& Balents, L. Quantum spin liquids: a review. Rep. Prog. Phys. 80, 016502 (2017).

2. Zhou, Y., Kanoda, K. \& Ng, T.-K. Quantum spin liquid states. Rev. Mod. Phys. 89 025003 (2017).

3. Balents, L. Spin liquids in frustrated magnets. Nature 464, 199-208 (2010).

4. Norman, M. R. Colloquium: Herbertsmithite and the search for the quantum spin liquid. Rev. Mod. Phys. 88, 041002 (2016).

5. Kitaev, A. Anyons in an exactly solved model and beyond. Ann. Phys. (N.Y.) 321, 2-111 (2006)

6. Hermanns, M., Kimchi, I. \& Knolle, J. Physics of the Kitaev model: fractionalization, dynamic correlations, and material connections. Annu. Rev. Condens. Matter Phys. 9, 17-33 (2018).

7. Kitaev, A. Y. Fault-tolerant quantum computation by anyons. Ann. Phys. (N.Y.) 303, 2-30 (2003).

8. Nayak, C., Simon, S. H., Stern, A., Freedman, M. \& Das Sarma, S. Non-abelian anyons and topological quantum computation. Rev. Mod. Phys. 80, 1083-1159 (2008).

9. Khaliullin, G. Orbital order and fluctuations in Mott insulators. Prog. Theor. Phys. Suppl. 160, 155-202 (2005).

10. Jackeli, G. \& Khaliullin, G. Mott insulators in the strong spin-orbit coupling limit: from Heisenberg to a quantum compass and Kitaev models. Phys. Rev. Lett. 102 017205 (2009).

11. Chaloupka, J., Jackeli, G. \& Khaliullin, G. Kitaev-Heisenberg model on a honeycomb lattice: possible exotic phases in iridium oxides $A_{2} \mathrm{IrO}_{3}$. Phys. Rev. Lett. 105, 027204 (2010).

12. Plumb, K. W. et al. $a-\mathrm{RuCl}_{3}$ : a spin-orbit assisted Mott insulator on a honeycomb lattice. Phys. Rev. B 90, 041112 (2014).

13. Winter, S. M. et al. Models and materials for generalized Kitaev magnetism. J. Phys. Condens. Matter 29, 493002 (2017).

14. Takagi, H., Takayama, T., Jackeli, G., Khaliullin, G. \& Nagler, S. E. Concept and realization of Kitaev quantum spin liquids. Nat. Rev. Phys 1, 264-280 (2019).

15. Liu, X. et al. Long-range magnetic ordering in $\mathrm{Na}_{2} \mid \mathrm{rO}_{2}$. Phys. Rev. B 83, 220403 (2011).

16. Choi, S. K. et al. Spin waves and revised crystal structure of honeycomb iridate $\mathrm{Na}_{2} \mathrm{IrO}_{3}$. Phys. Rev. Lett. 108, 127204 (2012).

17. $\mathrm{Ye}, \mathrm{F}$. et al. Direct evidence of a zigzag spin-chain structure in the honeycomb lattice: a neutron and x-ray diffraction investigation of single-crystal $\mathrm{Na}_{2} \mid \mathrm{IO}_{2}$. Phys. Rev. B 85, 180403 (2012).

18. Sears, J. A. et al. Magnetic order in $a-\mathrm{RuCl}_{3}$ : a honeycomb-lattice quantum magnet with strong spin-orbit coupling. Phys. Rev. B 91, 144420 (2015).
19. Johnson, R. D. et al. Monoclinic crystal structure of $a-\mathrm{RuCl}_{3}$ and the zigzag antiferromagnetic ground state. Phys. Rev. B 92, 235119 (2015).

20. Cao, H. B. et al. Low-temperature crystal and magnetic structure of $a-\mathrm{RuCl}_{3}$. Phys. Rev. B 93, 134423 (2016)

21. Banerjee, A. et al. Proximate Kitaev quantum spin liquid behaviour in a honeycomb magnet. Nat. Mat. 15, 733-740 (2016).

22. Williams, S. C. et al. Incommensurate counterrotating magnetic order stabilized by Kitaev interactions in the layered honeycomb $\alpha$-Li $\mathrm{Li}_{2} \mathrm{IO}_{3}$. Phys. Rev. B 93, 195158 (2016).

23. Banerjee, A. et al. Neutron scattering in the proximate quantum spin liquid

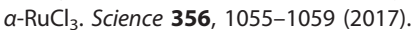

24. Banerjee, A. et al. Excitations in the field-induced quantum spin liquid state of a-RuCl. npj Quantum Mater 3, 8 (2018).

25. Do, S.-H. et al. Majorana fermions in the Kitaev quantum spin system $a-\mathrm{RuCl}_{3}$. Nat. Phys. 13, 1079-1084 (2017).

26. Ran, K. et al. Spin-wave excitations evidencing the Kitaev interaction in single crystalline $a-\mathrm{RuCl}_{3}$. Phys. Rev. Lett. 118, 107203 (2017).

27. Winter, S. M. et al. Breakdown of magnons in a strongly spin-orbital coupled magnet. Nat. Commun. 8, 1152 (2017).

28. Kasahara, Y. et al. Majorana quantization and half-integer thermal quantum Hall effect in a Kitaev spin liquid. Nature 559, 227-231 (2018).

29. Zheng, J. et al. Gapless spin excitations in the field-induced quantum spin liquid phase of $a-\mathrm{RuCl}_{3}$. Phys. Rev. Lett. 119, 227208 (2017).

30. Baek, S.-H. et al. Evidence for a field-induced quantum spin liquid in $a-\mathrm{RuCl}_{3}$. Phys. Rev. Lett. 119, 037201 (2017).

31. Sears, J. A., Zhao, Y., Xu, Z., Lynn, J. W. \& Kim, Y.-J. Phase diagram of $a-\mathrm{RuCl}_{3}$ in an in-plane magnetic field. Phys. Rev. B 95, 180411 (2017).

32. Balz, C. et al. Finite field regime for a quantum spin liquid in $a-\mathrm{RuCl}_{3}$. Phys. Rev. $B$ 100, 060405 (2019)

33. Wolter, A. U. B. et al. Field-induced quantum criticality in the Kitaev system a-RuCl . Phys. Rev. B 96, 041405 (2017).

34. Widmann, S. et al. Thermodynamic evidence of fractionalized excitations in a-RuCl. Phys. Rev. B 99, 094415 (2019).

35. Janša, N. et al. Observation of two types of fractional excitation in the Kitaev honeycomb magnet. Nat. Phys. 14, 786-790 (2018).

36. Wellm, C. et al. Signatures of low-energy fractionalized excitations in $a-\mathrm{RuCl}_{3}$ from field-dependent microwave absorption. Phys. Rev. B 98, 184408 (2018).

37. Sandilands, L. J., Tian, Y., Plumb, K. W., Kim, Y.-J. \& Burch, K. S. Scattering continuum and possible fractionalized excitations in $a-\mathrm{RuCl}_{3}$. Phys. Rev. Lett. 114, 147201 (2015).

38. $\mathrm{Du}, \mathrm{L}$. et al. $2 \mathrm{D}$ proximate quantum spin liquid state in atomic-thin $a-\mathrm{RuCl}_{3} .2 D$ Mater 6, 015014 (2018).

39. Wang, Y. et al. High temperature Fermi statistics from Majorana fermions in an insulating magnet. Preprint at https://arxiv.org/abs/1809.07782 (2018).

40. $\mathrm{Wu}$, L. et al. Field evolution of magnons in $\alpha-\mathrm{RuCl}_{3}$ by high-resolution polarized terahertz spectroscopy. Phys. Rev. B 98, 094425 (2018).

41. Reschke, S. et al. Terahertz excitations in $a-\mathrm{RuCl}_{3}$ : Majorana fermions and rigidplane shear and compression modes. Phys. Rev. B 100, 100403 (2019).

42. Little, A. et al. Antiferromagnetic resonance and terahertz continuum in $a-\mathrm{RuCl}_{3}$. Phys. Rev. Lett. 119, 227201 (2017).

43. Wang, Z. et al. Magnetic excitations and continuum of a possibly field-induced quantum spin liquid in $a-\mathrm{RuCl}_{3}$. Phys. Rev. Lett. 119, 227202 (2017).

44. Eichstaedt, $C$. et al. Deriving models for the Kitaev spin-liquid candidate material $a-\mathrm{RuCl}_{3}$ from first principles. Phys. Rev. B 100, 075110 (2019).

45. Kim, H.-S., V, V. S., Catuneanu, A. \& Kee, H.-Y. Kitaev magnetism in honeycomb $\mathrm{RuCl}_{3}$ with intermediate spin-orbit coupling. Phys. Rev. B 91, 241110 (2015).

46. Kim, H.-S. \& Kee, H.-Y. Crystal structure and magnetism in $a-\mathrm{RuCl}_{3}$ : An ab initio study. Phys. Rev. B 93, 155143 (2016).

47. Winter, S. M., Li, Y., Jeschke, H. O. \& Valentí, R. Challenges in design of Kitaev materials: magnetic interactions from competing energy scales. Phys. Rev. B 93, 214431 (2016).

48. Yadav, R. et al. Kitaev exchange and field-induced quantum spin-liquid states in honeycomb a-RuCl . Sci. Rep. 6, 37925 (2016).

49. Hou, Y. S., Xiang, H. J. \& Gong, X. G. Unveiling magnetic interactions of ruthenium trichloride via constraining direction of orbital moments: potential routes to realize a quantum spin liquid. Phys. Rev. B 96, 054410 (2017).

50. Wang, W., Dong, Z.-Y., Yu, S.-L. \& Li, J.-X. Theoretical investigation of magnetic dynamics in $a-\mathrm{RuCl}_{3}$. Phys. Rev. B 96, 115103 (2017).

51. Winter, S. M., Riedl, K., Kaib, D., Coldea, R. \& Valentí, R. Probing $a-\mathrm{RuCl}_{3}$ beyond magnetic order: effects of temperature and magnetic field. Phys. Rev. Lett. 120, 077203 (2018)

52. Cookmeyer, J. \& Moore, J. E. Spin-wave analysis of the low-temperature thermal Hall effect in the candidate Kitaev spin liquid $a$-RuCl 3 . Phys. Rev. B 98, 060412 (2018). 
53. Suzuki, T. \& Suga, S.-i. Effective model with strong Kitaev interactions for $a-\mathrm{RuCl}_{3}$. Phys. Rev. B 97, 134424 (2018).

54. Dagotto, E. Correlated electrons in high-temperature superconductors. Rev. Mod. Phys. 66, 763-840 (1994).

55. Sugiura, S. \& Shimizu, A. Thermal pure quantum states at finite temperature. Phys. Rev. Lett. 108, 240401 (2012).

56. Suzuki, T. \& Suga, S.-i. Dynamical spin structure factors of $a-\mathrm{RuCl}_{3}$. J. Phys. Conf. Ser. 969, 012123 (2018).

57. Rau, J. G., Lee, E. K.-H. \& Kee, H.-Y. Generic spin model for the honeycomb iridates beyond the Kitaev limit. Phys. Rev. Lett. 112, 077204 (2014).

58. Rau, J. G. \& Kee, H.-Y. Trigonal distortion in the honeycomb iridates: Proximity of zigzag and spiral phases in $\mathrm{Na}_{2} \mathrm{IrO}_{3}$. Preprint at https://arxiv.org/abs/1408.4811 (2014).

59. Rusnačko, J., Gotfryd, D. \& Chaloupka, J. Kitaev-like honeycomb magnets: global phase behavior and emergent effective models. Phys. Rev. B 99, 064425 (2019).

60. Knolle, J., Kovrizhin, D. L., Chalker, J. T. \& Moessner, R. Dynamics of a twodimensional quantum spin liquid: Signatures of emergent Majorana fermions and fluxes. Phys. Rev. Lett. 112, 207203 (2014).

61. Nasu, J., Udagawa, M. \& Motome, Y. Vaporization of Kitaev spin liquids. Phys. Rev. Lett. 113, 197205 (2014).

62. Nasu, J., Udagawa, M. \& Motome, Y. Thermal fractionalization of quantum spins in a Kitaev model: temperature-linear specific heat and coherent transport of Majorana fermions. Phys. Rev. B 92, 115122 (2015).

63. Yamaji, Y. et al. Clues and criteria for designing a Kitaev spin liquid revealed by thermal and spin excitations of the honeycomb iridate $\mathrm{Na}_{2} \mathrm{IrO}_{3}$. Phys. Rev. B 93, 174425 (2016).

64. Catuneanu, A., Yamaji, Y., Wachtel, G., Kim, Y. B. \& Kee, H.-Y. Path to stable quantum spin liquids in spin-orbit coupled correlated materials. npj Quantum Mater 3, 23 (2018).

65. Hardy, V., Lambert, S., Lees, M. R. \& McK. Paul, D. Specific heat and magnetization study on single crystals of the frustrated quasi-one-dimensional oxide $\mathrm{Ca}_{3} \mathrm{Co}_{2} \mathrm{O}_{6}$. Phys. Rev. B 68, 014424 (2003).

66. Samarakoon, A. M. et al. Classical and quantum spin dynamics of the honeycomb $\Gamma$ model. Phys. Rev. B 98, 045121 (2018).

67. Hirobe, D., Sato, M., Shiomi, Y., Tanaka, H. \& Saitoh, E. Magnetic thermal conductivity far above the Néel temperature in the Kitaev-magnet candidate $a-\mathrm{RuCl}_{3}$. Phys. Rev. B 95, 241112 (2017).

68. Kubota, Y., Tanaka, H., Ono, T., Narumi, Y. \& Kindo, K. Successive magnetic phase transitions in $a-\mathrm{RuCl}_{3}: \mathrm{XY}$-like frustrated magnet on the honeycomb lattice. Phys. Rev. B 91, 094422 (2015).

69. Sandilands, L. J. et al. Optical probe of Heisenberg-Kitaev magnetism in $a-\mathrm{RuCl}_{3}$. Phys. Rev. B 94, 195156 (2016).

70. Lampen-Kelley, P. et al. Anisotropic susceptibilities in the honeycomb Kitaev system $a-\mathrm{RuCl}_{3}$. Phys. Rev. B 98, 100403 (2018).

71. Gordon, J. S., Catuneanu, A., Sørensen, E. S. \& Kee, H.-Y. Theory of the fieldrevealed Kitaev spin liquid. Nat. Comms. 10, 2470 (2019).

72. Takikawa, D. \& Fujimoto, S. Impact of off-diagonal exchange interactions on the Kitaev spin-liquid state of $a-\mathrm{RuCl}_{3}$. Phys. Rev. B 99, 224409 (2019).

73. Kawamura, M. et al. Quantum lattice model solver $\mathcal{H} \phi$. Comp. Phys. Comms. 217, 180-192 (2017).

74. Sugiura, S. Formulation of Statistical Mechanics Based on Thermal Pure Quantum States. (Springer, Singapore, 2017).

75. Chaloupka, J. \& Khaliullin, G. Magnetic anisotropy in the Kitaev model systems $\mathrm{Na}_{2} \mathrm{IrO}_{3}$ and $a-\mathrm{RuCl}_{3}$. Phys. Rev. B 94, 064435 (2016).

76. Majumder, M. et al. Anisotropic $R u^{3+} 4 d^{5}$ magnetism in the $a-\mathrm{RuCl}_{3}$ honeycomb system: Susceptibility, specific heat, and zero-field NMR. Phys. Rev. B 91, 180401 (2015).

77. Agrestini, S. et al. Electronically highly cubic conditions for Ru in $a-\mathrm{RuCl}_{3}$. Phys. Rev. B 96, 161107 (2017).

78. Modic, K. A., Ramshaw, B. J., Shekhter, A. \& Varma, C. M. Chiral spin order in some purported Kitaev spin-liquid compounds. Phys. Rev. B 98, 205110 (2018).

79. Suzuki, T. \& Suga S.-i. Erratum: Effective model with strong Kitaev interactions for a-RuCl 3 [Phys. Rev. B 97, 134424 (2018)]. Phys. Rev. B 99, 249902 (2019).

80. Janssen, L., Andrade, E. C. \& Vojta, M. Magnetization processes of zigzag states on the honeycomb lattice: Identifying spin models for $a-\mathrm{RuCl}_{3}$ and $\mathrm{Na}_{2} \mid \mathrm{IO}_{3}$. Phys. Rev. B 96, 064430 (2017).
81. Eichstaedt, C. et al. Deriving models for the Kitaev spin-liquid candidate material

$a-\mathrm{RuCl}_{3}$ from first principles. Preprint at https://arxiv.org/abs/1904.01523v1 (2019).

82. Ozel, I. O. et al. Magnetic field-dependent low-energy magnon dynamics in $a-\mathrm{RuCl}_{3}$. Phys. Rev. B 100, 085108 (2019).

\section{ACKNOWLEDGEMENTS}

We thank C. Balz, A. Banerjee, T. Berlijn, S. E. Nagler, A. M. Samarakoon, and D. A. Tennant for helpful discussions, and A. Loidl for providing the magnetic specific data. We thank Y. Yamaji both for useful discussions and assistance with $\mathcal{H} \Phi$.

The research by P.L. and S.O. was supported by the Scientific Discovery through Advanced Computing (SciDAC) program funded by the US Department of Energy, Office of Science, Advanced Scientific Computing Research and Basic Energy Sciences, Division of Materials Sciences and Engineering. This research used resources of the Oak Ridge Leadership Computing Facility, which is a DOE Office of Science User Facility supported under Contract DE-AC05-00OR22725, and of the Compute and Data Environment for Science (CADES) at the Oak Ridge National Laboratory, which is managed by UT-Battelle and supported by the Office of Science of the U.S. Department of Energy under Contract No. DE-AC05-00OR22725. An award of computer time was provided by the INCITE program. A portion of the work was conducted at the Center for Nanophase Materials Sciences, which is a DOE Office of Science User Facility.

\section{AUTHOR CONTRIBUTIONS}

P.L. performed the ED and TPQ calculations. S.O. supervised the study. Both authors contributed to the writing of the manuscript.

\section{COMPETING INTERESTS}

The authors declare no competing interests.

\section{ADDITIONAL INFORMATION}

Supplementary information is available for this paper at https://doi.org/10.1038/ s41535-019-0203-y.

Correspondence and requests for materials should be addressed to P.L. or S.O.

Reprints and permission information is available at http://www.nature.com/ reprints

Publisher's note Springer Nature remains neutral with regard to jurisdictional claims in published maps and institutional affiliations.

Open Access This article is licensed under a Creative Commons Attribution 4.0 International License, which permits use, sharing, adaptation, distribution and reproduction in any medium or format, as long as you give appropriate credit to the original author(s) and the source, provide a link to the Creative Commons license, and indicate if changes were made. The images or other third party material in this article are included in the article's Creative Commons license, unless indicated otherwise in a credit line to the material. If material is not included in the article's Creative Commons license and your intended use is not permitted by statutory regulation or exceeds the permitted use, you will need to obtain permission directly from the copyright holder. To view a copy of this license, visit http://creativecommons. org/licenses/by/4.0/.

This is a U.S. government work and not under copyright protection in the U.S.; foreign copyright protection may apply 2020 